\title{
Biosynthesis of Thiamin in Bacillus subtilis. Isolation of Mutants Accumulating 4-Amino-5-hydroxymethyl- 2-methylpyrimidine Phosphate
}

\author{
By W. WALTER AND A. BACHER \\ Institut für Mikrobiologie, Universität Hohenheim, 7000 Stuttgart 70, \\ German Federal Republic
}

(Received 16 May 1977; revised I6 July 1977)

\begin{abstract}
Thiamin-deficient mutants of Bacillus subtilis were characterized by their growth responses to the pyrimidine and thiazole moieties of the vitamin molecule and by cross-feeding tests. All mutants growing on the thiazole moiety and all mutants with an absolute requirement for thiamin fed all those growing on the pyrimidine moiety. No other cross-feeding effects were observed. From the culture fluid of a mutant growing on the thiazole moiety, two compounds were isolated which supported growth of mutants requiring the pyrimidine moiety. These compounds were identified by chromatographic, bioautographic and spectrophotometric procedures as 4-amino-5-hydroxymethyl-2-methylpyrimidine and its monophosphate derivative.
\end{abstract}

\section{INTRODUCTION}

The enzymic formation of thiamin from 4-amino-5-hydroxymethyl-2-methylpyrimidine (which, throughout this paper, will be termed hydroxymethylpyrimidine) and 5 - $(\beta$-hydroxyethyl)-4-methylthiazole ( = thiazole) has been studied in considerable detail in various microorganisms. Hydroxymethylpyrimidine is converted to the pyrophosphate by the subsequent action of two phosphokinases (Camiener \& Brown, I960a,b). Thiazole is converted to the monophosphate. The two phosphoric acid esters are condensed with formation of thiamin monophosphate (Leder, 1959). Thiamin monophosphate is converted to the pyrophosphate by direct phosphorylation in Escherichia coli and in Micrococcus sp. (Nishino, Iwashima \& Nose, I97I ; Sanemori, Egi \& Kawasaki, 1976). In yeast, however, thiamin monophosphate is hydrolysed to free thiamin which is subsequently converted to the pyrophosphate by thiamin pyrophosphokinase (Camiener \& Brown, 1959).

The basic pathways leading to the pyrimidine and thiazole moieties of the vitamin molecule are incompletely understood. Isotope incorporation studies with a variety of basic metabolites in various organisms yielded conflicting results. The extensive literature has been reviewed by Brown (1972).

The frequent occurrence of pleiotropic Ath- mutants requiring both adenine and thiamin led Newell \& Tucker (1968 $a, b)$ to the concept of a basic common pathway for the biosynthesis of these metabolites. The thiamin requirement of an Ath- mutant of Salmonella typhimurium was satisfied by the purine precursor, 4-aminoimidazole riboside phosphate. Newell \& Tucker proposed that this compound is a precursor of the pyrimidine moiety of thiamin.

Nakayama (1956) found that mutants of $E$. coli deficient in the pyrimidine moiety of thiamin can grow on hydroxymethylpyrimidine and also on its 5 -formyl and 5-aminomethyl analogues. All three compounds and the 5-methoxymethyl analogue were isolated from the 
culture fluid of a thiamin-deficient mutant of Neurospora crassa (Diorio \& Lewin, I $968 a, b$ ). Wei \& Lewin (1970, 197I) described the conversion of 4-amino-5-aminomethyl-2methylpyrimidine (=aminomethylpyrimidine) and 4-amino-5-formyl-2-methylpyrimidine (=formylpyrimidine) to hydroxymethylpyrimidine by cell extracts of Saccharomyces cerevisiae.

Two unlinked thi genes have been mapped on the chromosome of Bacillus subtilis (Young \& Wilson, 1975), but the mutants have not been characterized biochemically. The present paper describes the isolation and biochemical characterization of thiamin-deficient mutants of $B$. subtilis. A mutant requiring thiazole for growth was found to excrete hydroxymethylpyrimidine phosphate.

\section{METHODS}

Chemicals. Hydroxymethylpyrimidine, aminomethylpyrimidine and thiazole were kindly provided by Hoffmann-La Roche, Basle, Switzerland. 4-Amino-5-chloromethyl-2-methylpyrimidine (=chloromethylpyrimidine) was a gift from BASF, Ludwigshafen, W. Germany. Thiamin, thiamin pyrophosphate and charcoal $(\mathrm{r} \cdot 5 \mathrm{~mm})$ were purchased from Merck; Dowex I-X8 and Dowex $50 \mathrm{~W}-\mathrm{X} 8$ from Serva, Malsch, W. Germany; thiamin monophosphate from Fluka, Buchs, Switzerland; AG I-X8 from Biorad; and alkaline phosphatase from Boehringer.

5 -( $\beta$-Hydroxyethyl)-4-methylthiazole 2 -phosphate (=thiazole phosphate) was prepared according to Lohmann \& Schuster (1937). 4-Amino-5-hydroxymethyl-2-methylpyrimidine phosphate (=hydroxymethylpyrimidine phosphate) was prepared from chloromethylpyrimidine according to Lewin \& Brown (1963). The compound was purified by chromatography on a column of Dowex I-X8 (formate form, $2 \times 20 \mathrm{~cm}$, elution with a gradient of 0 to $0.4 \mathrm{M}$-ammonium formate).

Media. Complete medium contained (g per 1 deionized water): nutrient broth (Difco), 8.0; yeast extract (Oxoid), 3.0; glucose, 3•0. The minimal medium was Spizizen's medium (Spizizen, 1958) supplemented with L-tryptophan ( $50 \mathrm{mg} \mathrm{l}^{-1}$ ). The medium was further supplemented as required in each experiment.

Strains. Bacillus subtilis I68M, trpC2, was kindly provided by Dr C. Anagnostopoulos, Gif sur Yvette. Thiamin-deficient mutants are described in Results.

Isolation of mutants. Bacillus subtilis $168 \mathrm{M}$ was harvested in the early exponential phase of growth and suspended in citrate/phosphate buffer pH 7. $N$-Methyl- $N^{\prime}$-nitro- $N$-nitrosoguanidine was added to a final concentration of $20 \mathrm{mg} \mathrm{l}^{-1}$ and the suspension was incubated at $37^{\circ} \mathrm{C}$ for $40 \mathrm{~min}$. The bacteria were then pelleted, washed, and resuspended in complete medium supplemented with adenine $\left(50 \mathrm{mg} \mathrm{l}^{-1}\right)$ and thiamin pyrophosphate $\left(\mathrm{I} \mathrm{mg} \mathrm{1}^{-1}\right)$. The suspension was incubated overnight with shaking. Bacteria were plated on complete medium supplemented with adenine $\left(50 \mathrm{mg} \mathrm{l}^{-1}\right)$. Colonies were replicated on minimal medium

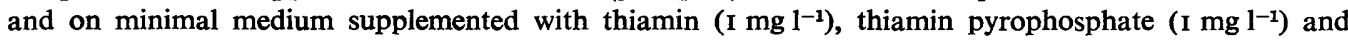
adenine (50 $\mathrm{mg} \mathrm{l}^{-1}$ ).

Growth tests. Approximately $10^{7}$ washed bacteria from an overnight culture were inoculated into flasks containing $20 \mathrm{ml}$ minimal medium supplemented as required, and incubated at $37^{\circ} \mathrm{C}$ with shaking. Turbidity $(623 \mathrm{~nm})$ was measured after $20 \mathrm{~h}$.

Cross-feeding tests. Method A. Approximately $10^{8}$ washed bacteria from an overnight culture were suspended in $20 \mathrm{ml}$ minimal agar and plated. Suspensions of tester strains $\left(10^{8}\right.$ bacteria $\left.\mathrm{ml}^{-1}\right)$ were then streaked on the plates. Growth of streaks was examined after 2 days.

Method B. Feeder mutants were grown in complete medium, washed, and resuspended in minimal medium. The suspensions were incubated overnight and then the culture fluid was separated and filter-sterilized. Portions were added to flasks containing $20 \mathrm{ml}$ minimal medium. The flasks were heatsterilized and inoculated with $10^{7}$ washed bacteria of the tester strains. Turbidity was monitored after $20 \mathrm{~h}$ incubation.

Bioautography. Method A. Paper chromatograms were placed on minimal agar plates containing the tester strain $\left(10^{8}\right.$ bacteria $\left.\mathrm{ml}^{-1}\right)$. After $90 \mathrm{~min}$, the chromatograms were removed, and the plates were incubated overnight.

Method B. Chromatograms were cut, and the strips were soaked in $20 \mathrm{ml}$ minimal medium for $2 \mathrm{~h}$. The medium was sterilized and inoculated with the tester strain as described under growth tests.

Reversion. Approximately $10^{9}$ bacteria were plated on minimal medium supplemented as required. Colonies were examined after 2 days.

Fermentation. Mutant T57 was grown in 101 minimal medium supplemented with thiamin $\left(30 \mu \mathrm{g}^{-1}\right)$ at $37^{\circ} \mathrm{C}$ with stirring ( $300 \mathrm{rev}$. $\mathrm{min}^{-1}$ ) and aeration $\left(1801 \mathrm{~h} \mathrm{~h}^{-1}\right)$. Bacteria were harvested after $24 \mathrm{~h}$ and resuspended in 101 of thiamin-free minimal medium. The suspension was incubated for $24 \mathrm{~h}$ as described above and the bacteria were removed by centrifugation. 
Table I. Growth requirements of the 53 thiamin-deficient mutants isolated

$\begin{aligned} \text { No. of mutants } & \text { Growth requirement } \\ \text { 2I } & \text { Thiamin } \\ 8 & \text { Hydroxymethylpyrimidine } \\ \text { I } 8 & \text { Thiazole } \\ 3 & \text { Hydroxymethylpyrimidine and thiazole } \\ 3 & \text { Hydroxymethylpyrimidine or thiazole }\end{aligned}$

Table 2. Cross-feeding experiments with B. subtilis mutants

The requirements of feeder and tester mutants were: $B_{1}$, thiamin; $T$, thiazole; $P$, hydroxymethylpyrimidine. The numbers of mutants examined are indicated in parentheses.

\begin{tabular}{lccc} 
& \multicolumn{3}{c}{ Tester mutants } \\
Feeder mutants & $\overbrace{\mathrm{B}_{1}(\mathrm{I} 7)}^{\mathrm{T}(\mathrm{I} 7)}$ & $\mathbf{P}(4)$ \\
$\mathbf{B}_{\mathbf{1}}(\mathrm{I} 7)$ & - & - & + \\
$\mathbf{T}(\mathrm{I})$ & - & - & + \\
$\mathbf{P}(4)$ & - & - & - \\
&,+ Growth; - , no growth. &
\end{tabular}

RESULTS

Mutants of Bacillus subtilis were obtained after treatment of strain I68M with $N$-methyl- $N^{\prime}-$ nitro- $N$-nitrosoguanidine in four independent mutation experiments. In a total of 53 mutants growing on thiamin and 224 mutants growing on adenine, no Ath- mutants, with simultaneous requirement for both thiamin and adenine, were obtained. Similarly, no mutants with a specific requirement for thiamin pyrophosphate were found.

The thiamin-deficient mutants were further characterized by their growth responses to hydroxymethylpyrimidine and thiazole (Table I). All mutants growing on hydroxymethylpyrimidine were also able to grow on the 5-aminomethyl and the 5-chloromethyl analogues and on hydroxymethylpyrimidine phosphate. Twenty-one mutants were unable to grow on hydroxymethylpyrimidine, thiazole or the corresponding phosphoric acid esters.

Three mutants required the simultaneous presence of hydroxymethylpyrimidine and thiazole for growth. These mutants were analysed by reversion tests. No spontaneous prototrophic revertants were obtained from any of them. However, all three mutants spontaneously segregated revertants growing on hydroxymethylpyrimidine in the absence of thiazole at a frequency of $10^{-8}$. Revertants growing on thiazole in the absence of hydroxymethylpyrimidine were also segregated at a similar frequency. We conclude that the mutants had acquired genetic defects in two different thiamin genes.

Three mutants could grow on either hydroxymethylpyrimidine or thiazole. However, further analysis showed that they could also grow on various amino acids (glycine, serine, phenylalanine, threonine, isoleucine, methionine) in the absence of any specific thiamin precursor. The basis for this behaviour is not understood.

The single-step mutants with a clearcut requirement for thiamin or one of the supposed precursors mentioned were studied by cross-feeding tests in several combinations (Table 2). All mutants with an absolute requirement for thiamin were able to feed those with a requirement for hydroxymethylpyrimidine. Similarly, all thiazole-requiring mutants fed the hydroxymethylpyrimidine-requiring mutants. No other cross-feeding phenomena were observed. In particular, the thiazole-deficient mutants did not respond to metabolic products from any strain studied. From these data, we tentatively concluded that the thiazole-deficient mutants and those with an absolute requirement for thiamin produce and excrete one or more compounds capable of replacing the pyrimidine moiety of thiamin. Subsequent studies were concerned with the isolation and identification of these compounds. 


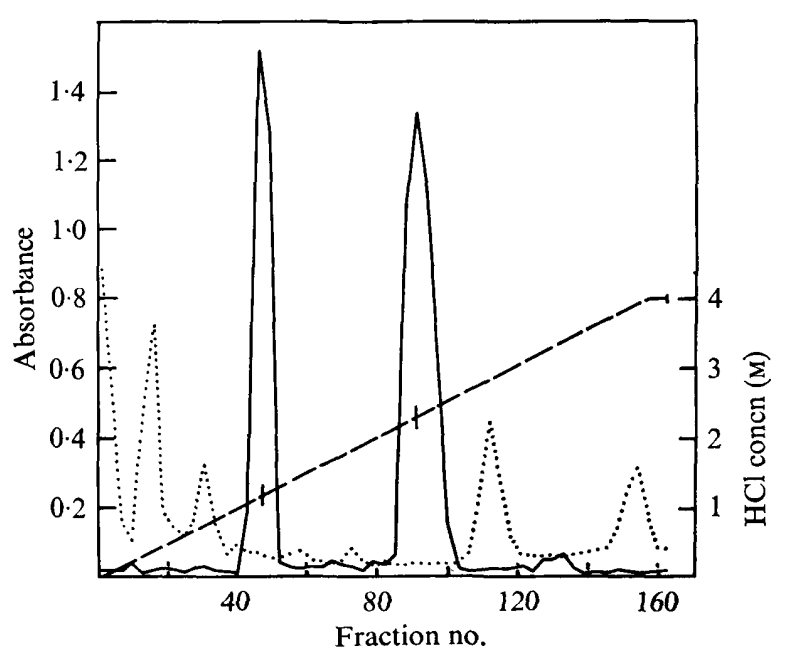

Fig. I. Chromatography of metabolites from B. subtilis mutant T57 (thiazole-deficient) on a column of Dowex $50 \mathrm{~W}-\mathrm{X} 8\left(\mathrm{H}^{+}\right.$form, $\mathrm{I} \cdot 7 \times 67 \mathrm{~cm}$, elution with a gradient of $\left.\mathrm{HCl}\right)$. Bioautography $\left(E_{623}\right),-; E_{245}, \cdots \cdots$; concentration of $\mathrm{HCl},---$.

The thiazole-requiring mutant 557 was grown in minimal medium supplemented with thiamin. The bacteria were harvested, resuspended in thiamin-free medium, and incubated overnight. Samples were taken at the beginning and at the end of the incubation period. These suspensions were sterilized and analysed for growth-promoting material by bioassay with the hydroxymethylpyrimidine-deficient mutant $\mathrm{T} 6$. We found that the amount of such material increased by a factor of 5 during incubation of mutant T57 in minimal medium, thus indicating its de novo formation. The amount of growth-promoting material produced corresponded to $0.15 \mathrm{mg}$ thiamin equivalents per 1 culture medium.

Accumulation experiments with mutant $\mathrm{T} 57$ were performed in 101 batches as described in Methods. The cell-free culture fluid was passed through a column of charcoal $(8 \times I 2 \mathrm{~cm})$. The column was washed with water and the growth-promoting material was eluted with ethanol/water ( $\mathrm{I}: \mathrm{I}, \mathrm{v} / \mathrm{v} ; 2 \mathrm{l})$. The elution was followed by bioassay with mutant $\mathrm{T} 6$. The recovery of growth-promoting material was $90 \%$. The eluate was taken to dryness under reduced pressure and the residue was dissolved in $3 \mathrm{ml}$ deionized water. The solution was placed on a column of Dowex $50 \mathrm{~W}-\mathrm{X} 8\left(\mathrm{H}^{+}\right.$form, $\left.\mathrm{I} \cdot 7 \times 67 \mathrm{~cm}\right)$ which was developed with a linear gradient of o to $4 \mathrm{M}-\mathrm{HCl}$ (total volume, 2 1). Elution of thiamin metabolites was followed by bioassay. The elution pattern is shown in Fig. I. Two different fractions stimulating growth of the hydroxymethylpyrimidine-deficient mutant $\mathrm{T} 6$ were eluted by $\mathrm{I} \cdot 2$ and $2 \cdot 3$ $\mathrm{M}-\mathrm{HCl}$. These fractions were taken to dryness under reduced pressure.

The material eluted by $2 \cdot 3 \mathrm{M}-\mathrm{HCl}$ (compound I) was placed on a column of AG I-X8 (formate form, $2 \times 20 \mathrm{~cm}$ ). The column was developed with a linear gradient of 0 to $0.4 \mathrm{M}$ ammonium formate (total volume, $500 \mathrm{ml}$ ). The el'tion was followed by bioassay with mutant T6 (Fig. 2). A single peak of growth-promoting material was observed in the eluate, at 0.1 M-ammonium formate. The ultraviolet spectrum of compound I showed the characteristic features of a simple pyrimidine derivative $\left(\lambda_{\max }\right.$ in $0.1 \mathrm{M}-\mathrm{HCl}, 245 \mathrm{~nm} ; \lambda_{\max }$ in 0.1 $\mathrm{M}-\mathrm{NaOH}, 27 \mathrm{I}$ and $233 \mathrm{~nm}$ ). By chromatographic comparison with various pyrimidine derivatives, compound I was identified as hydroxymethylpyrimidine. Compound I showed the same elution patterns as an authentic sample of hydroxymethylpyrimidine on columns of Dowex 50W-X8 and AG I-X8, respectively (experimental conditions as described above). In addition, both compounds migrated the same distance on paper chromatograms (Table 3). 


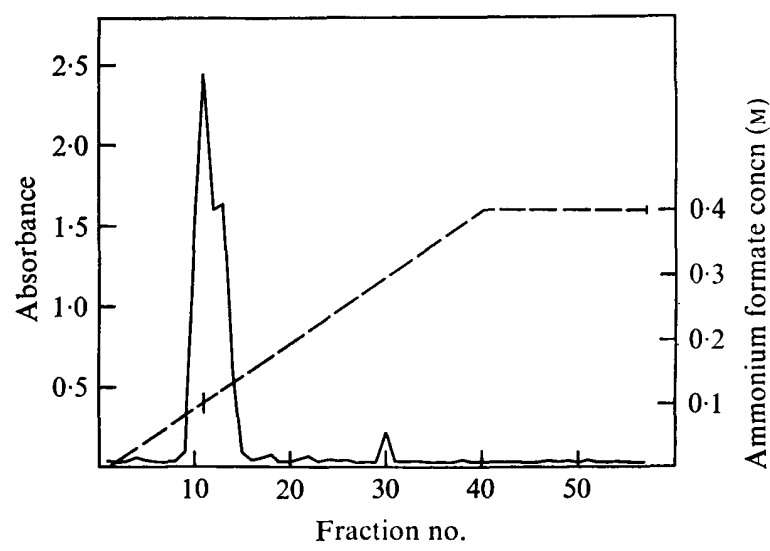

Fig. 2. Chromatography of compound I on a column of AG I-X8 (formate form, $2 \times 20 \mathrm{~cm}$, elution with a gradient of ammonium formate). Bioautography $\left(E_{623}\right),-$; concentration of eluant, ---.

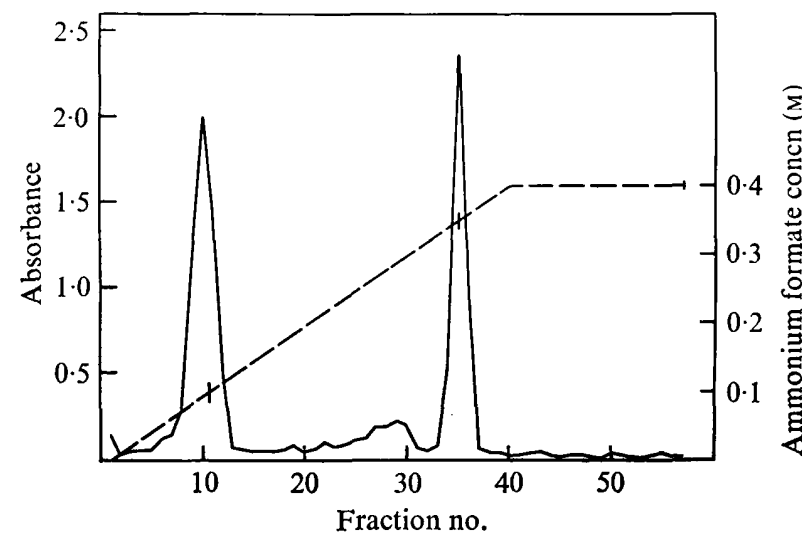

Fig. 3. Chromatography of compound 2 on a column of AG I-X8. Experimental conditions and key as in Fig. 2.

Further purification of compound 2 (eluted from Dowex $50 \mathrm{~W}-\mathrm{X} 8$ by $\mathrm{I} \cdot 2 \mathrm{M}-\mathrm{HCl}$, see above) was performed by chromatography on a column of AG I-X8 under the experimental conditions described above. Fractions with growth-promoting activity were eluted by $0 . \mathrm{I}$ and $0.35 \mathrm{M}$-ammonium formate (Fig. 3). When the material eluted by $0.35 \mathrm{M}$ ammonium formate was subsequently rechromatographed under the same conditions, the same pattern of two growth-promoting fractions eluted by $0 . \mathrm{I}$ and $0.35 \mathrm{M}$-ammonium formate was again observed. This indicates that compound 2 decomposes slowly under these conditions with formation of the material eluted by $0 \cdot 1 \mathrm{M}$-ammonium formate. The identity of the latter compound with hydroxymethylpyrimidine was shown by the procedures described above (Table 3). It follows that compound 2 is a derivative of hydroxymethylpyrimidine. The conversion of compound 2 to hydroxymethylpyrimidine is greatly accelerated by alkaline phosphatase. The hypothesis that compound 2 is the phosphoric acid ester of hydroxymethylpyrimidine was confirmed by comparison with an authentic sample of hydroxymethylpyrimidine phosphate. The compounds were identical with respect to (i) chromatography on columns of Dowex 50W-X8 and AG I-X8, (ii) paper chromatography (Table 3), (iii) hydrolysis by alkaline phosphatase, and (iv) ultraviolet spectra. 


\section{Table 3. Paper chromatography of accumulation products of $B$. subtilis mutant $\mathrm{T} 57$ and authentic compounds}

Compounds were spotted on Macherey \& Nagel paper MN 263 and developed by ascending chromatography. Spots were detected by bioautography as described in Methods. Solvent systems: I, 2-propanol/I M-HCl (I 7:8, v/v); 2, 2-propanol/acetic acid/water (I 70:4I:39, v/v).

$\begin{array}{lcc}\text { Compound } & \text { Solvent I } & \text { Solvent 2 } \\ \text { Thiamin } & 0.34 & 0.45 \\ \text { Thiamin phosphate } & 0.40 & 0.13 \\ \text { Thiamin pyrophosphate } & 0.22 & 0 \\ \text { Hydroxymethylpyrimidine } & 0.50 & 0.55 \\ \text { Hydroxymethylpyrimidine phosphate } & 0.52 & 0.18 \\ \text { Aminomethylpyrimidine } & 0.25 & 0.21 \\ \text { Compound I } & 0.48 & 0.52 \\ \text { Compound 2 } & 0.52 & 0.20 \\ \text { Degradation product of compound 2 } & 0.49 & 0.53\end{array}$

\section{DISCUSSION}

A total of 53 thiamin-deficient mutants of $B$. subtilis obtained in four mutation experiments have been characterized by feeding and accumulation tests. The results suggest the existence of considerable differences of mutant types among different microbial species.

Mutants with a double requirement for thiamin and purines ( $\mathrm{Ath}^{-}$) have been isolated from $S$. typhimurium (Yura, I956) and $E$. coli (Stouthamer, de Haan \& Nijkamp, I965). In the latter species, two ath loci were assigned to early steps in the biosynthesis of purines. No Ath- mutants were found in studies on Sacch. cerevisiae (Müller-Falcke, I974). In the present work, attempts to isolate mutants with a double requirement for thiamin and adenine were unsuccessful although 224 adenine-deficient mutants and 53 thiamin-deficient mutants were isolated. Since the mutants were derived from a limited number of mutagenized cultures, the possibility remains that several isolates were descendants of the same original mutant. With this qualification, the material represents a minimum of 22 independent Thimutants. On this basis, we suggest tentatively that either Ath ${ }^{-}$mutants may not exist in $B$. subtilis or that they may have additional metabolic defects which interfere with their detection.

Mutants with a double requirement for the pyrimidine and thiazole moieties occur frequently in Sacch. cerevisiae and in N. crassa. In Sacch. cerevisiae, this phenotype can be produced by mutation in one of several unlinked genes (Müller-Falcke, I974). In the present study, all mutants of $B$. subtilis with a double requirement for the pyrimidine and the thiazole moieties were shown to be double mutants.

The cross-feeding experiments with the $B$. subtilis mutants showed a surprisingly simple pattern. Only one group of feeding strains comprising all mutants with an absolute requirement for thiamin and all mutants growing on thiazole was found. The feeder mutants were able to feed all mutants requiring the pyrimidine moiety. This suggested that the feeding mutants produce one or more compounds of the pyrimidine pathway which cannot be metabolized further due to the genetic defect. This was confirmed by the isolation, in pure form, of two compounds with growth-promoting activity from the culture fluid of a thiazoledeficient mutant. The de novo formation of these metabolites was ascertained by quantitative bioassays. The compounds were subsequently identified as hydroxymethylpyrimidine and its monophosphate derivative. The isolation of hydroxymethylpyrimidine phosphate from a microbial culture has not been reported previously, although the enzymic formation of the compound by a yeast enzyme is known (Camiener \& Brown, 1960a). No other growthpromoting factors were found. In particular, the accumulation of aminomethylpyrimidine 
was ruled out by a reconstruction experiment using the authentic compound. It is possible that the isolated hydroxymethylpyrimidine originates from the degradation of the monophosphate since we have found that the latter compound is rather unstable under our experimental conditions.

The pyrimidine-deficient mutants could not be subdivided by cross-feeding experiments. In particular, all of them were able to utilize chloromethylpyrimidine and aminomethylpyrimidine, i.e. no mutants had an absolute requirement for hydroxymethylpyrimidine.

Surprisingly, none of the thiazole-deficient mutants could be fed by any of the other mutants. This may indicate that thiazole is an intermediate in a salvage pathway rather than in the de novo synthesis of the vitamin (Harris, 1955, 1956). To the best of our knowledge, thiazole compounds have not been isolated from thiamin-deficient mutants.

This work was supported by the Deutsche Forschungsgemeinschaft. Professor F. Lingens kindly provided the facilities and support of his institute. The technical assistance of E. Gerbershagen is gratefully acknowledged.

\section{REFERENCES}

Brown, G. M. (1972). Thiamine. VII. Biogenesis. In The Vitamins, vol. 5, pp. 122-129. Edited by W. H. Sebrell, Jr, \& R. S. Harris. New York: Academic Press.

Camiener, G. W. \& Brown, G. M. (1959). The biosynthesis of thiamine and thiamine phosphates by extracts of baker's yeast. Journal of the American Chemical Society 8r, 3800.

Camiener, G. W. \& Brown, G. M. (I960a). The biosynthesis of thiamine. I. Enzymatic formation of thiamine and phosphate esters of the pyrimidine moiety of thiamine. Journal of Biological Chemistry 235, 2404-24I0.

Camiener, G. W. \& Brown, G. M. (1960 $b$ ). The biosynthesis of thiamine. II. Fractionation of enzyme system and identification of thiazole phosphate and thiamine-phosphate as intermediates. Journal of Biological Chemistry 235, 241 I-24I 7.

Diorio, A. F. \& LewIN, L. M. (I968a). The pathways of thiamine biosynthesis. Some factors controlling synthesis of the pyrimidine moiety by thiamine auxotrophs of Neurospora crassa. Journal of Biological Chemistry 243, 3999-4005.

Diorio, A. F. \& LEWIN, L. M. (1968b). The pathways of thiamine biosynthesis. Separation, purification and identification of some precursors of the pyrimidine moiety of thiamine produced by Neurospora crassa auxotrophs. Journal of Biological Chemistry 243, 4006-40I 2.

HARRIS, D. L. (1955). Alternative pathways of thiamine biosynthesis in Neurospora. Archives of Biochemistry and Biophysics 57, 240-25I.

HARRIS, D. L. (1956). Interaction of thiamine and pyridoxine in Neurospora. II. Competition between pyridoxine and the pyrimidine precursor of thiamine. Archives of Biochemistry and Biophysics 60, 35-43.

LEDER, I. G. (1959). The enzymatic synthesis of thiamine monophosphate. Biochemical and Biophysical Research Communications 1, 63-66.

LEWIN, L. M. \& Brown, G. M. (1963). The biosynthesis of thiamine. IV. Inhibition by vitamin $\mathrm{B}_{6}$ compounds. Archives of Biochemistry and Biophysics ror, 197-203.
LOHMANN, K. \& Schuster, P. (1937). Untersuchungen über die Cocarboxylase. Biochemische Zeitschrift 294, I 88-2 I4.

MüLlER-FALCKE, S. (1974). Genetische Untersuchungen an Thiaminmangelmutanten von Saccharomyces cerevisiae. Dissertation, Universität Hohenheim, Stuttgart, German Federal Republic.

NAKAYAMA, H. (1956). Thiamine auxotrophs of Escherichia coli. I. Nutritional activities of thiamine and related compounds. Vitamins (Kyoto) ro, 356-365.

Newell, P. C. \& Tucker, R. G. (1968a). Precursors of the pyrimidine moiety of thiamine. Biochemical Journal ro6, 27I-277.

Newell, P. C. \& Tucker, R. G. (1968b). Biosynthesis of the pyrimidine moiety of thiamine. A new route of pyrimidine biosynthesis involving purine intermediates. Biochemical Journal 106, 279-287.

Nishino, H., Iwashima, A. \& Nose, Y. (1971). Biogenesis of cocarboxylase in Escherichia coli: a novel enzyme catalyzing the formation of thiamine pyrophosphate from thiamine monophosphate. Biochemical and Biophysical Research Communications 45, 363-368.

SANemori, H., EgI, Y. \& Kawasaki, T. (1976). Pathway of thiamine pyrophosphate synthesis in Micrococcus denitrificans. Journal of Bacteriology I26, 1030-1036.

SpIZIZEN, J. (1958). Transformation of biochemically deficient strains of Bacillus subtilis by deoxyribonucleate. Proceedings of the National Academy of Sciences of the United States of America 44, 1072-1078.

Stouthamer, A. H., De HaAN, P. G. \& NiJkamp, H. J. J. (1965). Mapping of purine markers in Escherichia coli K12. Genetical Research 6, 422453.

WEI, R. \& LEWIN, L. M. (1970). The biosynthesis of thiamine. Conversion of 2-methyl-4-amino-5formylpyrimidine to 2-methyl-4-amino-5-hydroxymethylpyrimidine by cell-free extracts of baker's yeast. Biochimica et biophysica acta 201, 334339. 
WeI, R. \& LEWIN, L. M. (I97I). The biosynthesis of thiamine. A yeast enzyme system which converts 2-methyl-4-amino-5-aminomethylpyrimidine to 2methyl-4-amino-5-hydroxymethylpyrimidine. Biochimica et biophysica acta 230, 253-257.

Young, F. E. \& Wilson, G. A. (1975). Chromosomal map of Bacillus subtilis. In Spores VI, pp. 596-6r4. Edited by P. Gerhardt, R. N. Costilow \& H. L. Sadoff. Washington: American Society for Microbiology.

YURA, T. (1956). Evidence of non-identical alleles in purine-requiring mutants of Salmonella typhimurium. Carnegie Institution of Washington Publication 6r2, 63-75. 